\title{
Points on Algebraic Curves and the Parametrization Problem *
}

\author{
Erik Hillgarter and Franz Winkler \\ Institut für Mathematik and RISC-LINZ \\ Johannes Kepler Universität \\ A-4040 Linz, Austria
}

Abstract.

A plane algebraic curve is given as the zeros of a bivariate polynomial. However, this implicit representation is badly suited for many applications, for instance in computer aided geometric design. What we want in many of these applications is a rational parametrization of an algebraic curve. There are several approaches to deciding whether an algebraic curve is rationally parametrizable and if so computing such a parametrization. In all these approaches we ultimately need some simple points on the curve. The field in which we can find such points crucially influences the coefficients in the resulting parametrization. We show how to find simple points over some practically interesting fields. Consequently, we are able to decide whether an algebraic curve defined over the rational numbers can be parametrized over the rationals or the reals. Some of these ideas also apply to parametrization of surfaces. If in the term geometric reasoning we do not only include the process of proving or disproving geometric statements, but also the analysis and manipulation of geometric objects, then algorithms for parametrization play an important role in this wider view of geometric reasoning.

\section{The parametrization problem}

An algebraic curve $\mathcal{C}$ in the affine plane $\mathbb{A}^{2}(\mathbb{K})$ over the algebraically closed field $\mathbb{K}$ is defined as

$$
\mathcal{C}=\left\{(x, y) \in \mathbb{K}^{2} \mid f(x, y)=0\right\}
$$

where $f(x, y)$ is a bivariate square-free polynomial with coefficients in $\mathbb{K}$, called the defining polynomial of $\mathcal{C}$. Observe that the defining polynomial of a plane algebraic curve is determined up to multiplication by non-zero constants in $\mathbb{K}$. The curve $\mathcal{C}$ is irreducible iff its defining polynomial is irreducible. The degree of $\mathcal{C}, \operatorname{deg}(\mathcal{C})$, is simply the degree of the defining polynomial $f$.

\footnotetext{
* Partially supported by Österr. Fonds zur Förderung der wissenschaftlichen Forschung, Proj. HySaX, Nr. P11160-TEC and ÖAD, Proj. Acción Integrada 30/97.
} 
The singularity structure of an algebraic curve is not fully apparent in the affine plane, since the curve might have "singularities at infinity". So from time to time we will need to view an algebraic curve as an object in the projective plane $\mathbb{P}^{2}(\mathbb{K})$. The affine plane is embedded into the projective plane by identifying an affine point $(a, b)$ with the projective point $(a: b: 1)$. In addition to these affine points, the projective plane contains points at infinity, with projective coordinates $(a: b: 0)$, where $(a, b) \neq(0,0)$. A projective curve agrees with the corresponding affine curve, except that finitely many points at infinity are added. The points on the projective curve are the solutions of $F(x, y, z)=0$, where $F(x, y, z)$ is the homogenization of $f(x, y)$ w.r.t. the homogenizing variable $z$.

Some algebraic curves can also be represented parametrically, i.e. their points can be generated by rational functions

$$
x(t)=\frac{p_{1}(t)}{q_{1}(t)}, \quad y(t)=\frac{p_{2}(t)}{q_{2}(t)},
$$

in $\mathbb{K}(t)$, i.e. $p_{1}(t), p_{2}(t), q_{1}(t), q_{2}(t) \in \mathbb{K}[t]$. More precisely, we have the following definition.

Definition I.1: If the irreducible affine plane curve $\mathcal{C}$ is defined by $f(x, y) \in$ $\mathbb{K}[x, y], \mathbb{K}$ an algebraically closed field of characteristic 0 , then $\mathcal{P}(t)=(x(t), y(t))$ $\in \mathbb{K}(t) \times \mathbb{K}(t)$ is a rational parametrization of $\mathcal{C}$ iff, except for finitely many exceptions, every evaluation $\left(x\left(t_{0}\right), y\left(t_{0}\right)\right)$ at $t_{0} \in \mathbb{K}$ is a point on $\mathcal{C}$, and conversely almost every point on $\mathcal{C}$ is the result of evaluating the parametrization at some element of $\mathbb{K}$.

In this case $\mathcal{C}$ is called parametrizable or rational.

Equivalently, $\mathcal{P}(t)=(x(t), y(t))$ is a rational parametrization of $\mathcal{C}$ if $\mathcal{P}$ : $\mathbb{K} \longrightarrow \mathcal{C}$ is rational and not both $x(t)$ and $y(t)$ are constant. Furthermore, if $\mathcal{P}$ is birational we say that $\mathcal{P}(t)$ is a proper parametrization.

In computer aided geometric design (cagd) one usually requires that the algebraic curves are rational, see e.g. [3]. In fact, transformation methods between these two representations of algebraic curves of genus 0 are of great interest in cagd. This problem appears as one of the 10 most important problems in cagd in $[8]$.

The problem of deciding whether an algebraic curve over an algebraically closed field of characteristic 0 is rational was solved by Hilbert and Hurwitz more than 100 years ago [1]. In fact, they prove that if the rational curve $\mathcal{C}$ is defined by a polynomial $f(x, y) \in \mathbb{K}[x, y]$, where $\mathbb{K}$ is not necessarily algebraically closed, then $\mathcal{C}$ has a parametrization with coefficients in an algebraic extension field $\mathbb{L}$ of $\mathbb{K}$ with $[\mathbb{L}: \mathbb{K}] \leq 2$ and $\mathbb{L}=\mathbb{K}$ for $\operatorname{deg}(f)$ odd. The construction of such an "optimal" parametrization requires $\mathcal{O}(\operatorname{deg}(f))$ birational transformations. Every one of these transformations decreases the degree of the curve by 2 , and thus ultimately leads to either a line (in the odd degree case) or to an irreducible conic $\mathcal{C}_{2}$ defined over the same field as the original curve $\mathcal{C}$. This reduction 
process was abbreviated in [12] and actually the corresponding conic can simply be interpolated. Every point on the conic $\mathcal{C}_{2}$ corresponds rationally to a point on $\mathcal{C}$ and vice versa (with finitely many exceptions). So, if we can find a point of $\mathcal{C}_{2}$ in an extension field $\mathbb{L}$ of $\mathbb{K}$, then we can parametrize $\mathcal{C}_{2}$ and $\mathcal{C}$ with coefficients in $\mathbb{L}$.

In a geometric approach to the parametrization problem, we consider a linear system $\mathcal{L}$ of curves having prescribed multiplicities at the singular points of $\mathcal{C}$. In particular, it is reasonable (but not necessary) to consider a system $\mathcal{L}$ of curves of degree $\operatorname{deg}(\mathcal{C})-2$ and require that every singular point $P$ of $\mathcal{C}$ be a point on every curve in $\mathcal{L}$ with multiplicity at least $\operatorname{mult}_{P}(\mathcal{C})-1$. $\mathcal{L}$ is called the system of adjoint curves of $\mathcal{C}$. The dimension of $\mathcal{L}$ is $\operatorname{deg}(\mathcal{C})-2$. So by fixing $\operatorname{deg}(\mathcal{C})-3$ simple points on $\mathcal{C}$ to also be simple points on every curve in $\mathcal{L}$, we reduce the dimension of $\mathcal{L}$ to 1 , i.e. every curve in $\mathcal{L}$ will intersect $\mathcal{C}$ in the fixed points (the singularities and some fixed simple points of $\mathcal{C}$ ) and exactly 1 additional "free" simple intersection point on $\mathcal{C}$, which depends rationally on the free parameter of the system $\mathcal{L}$. Thus, the rational expression of the "free" intersection point immediately yields the desired parametrization of $\mathcal{C}$. This geometric approach was theoretically described in [14] and was investigated from the computer algebra point of view in [11].

Example I.1: The irreducible curve $\mathcal{C}$ in the affine plane over the complex numbers $\mathbb{C}$ is defined by

$$
\begin{gathered}
f(x, y)=x^{5}+3 x^{4} y-2 x^{3} y^{2}+x^{2} y^{3}+y^{5}-2 x^{4}-4 x^{3} y+x^{2} y^{2} \\
-x y^{3}-2 y^{4}+x^{3}+x^{2} y+x y^{2}+y^{3} .
\end{gathered}
$$

$\mathcal{C}$ has a triple point $P_{1}$ at the origin $(0,0)$, and double points $P_{2}=(0,1), P_{3}=$ $(1,1), P_{4}=(1,0)$. So the genus of $\mathcal{C}$ is 0 , and it is parametrizable. In order to construct a subsystem of dimension 1 of the system of adjoints of $\mathcal{C}$, we need 2 simple points on $\mathcal{C}$. Intersecting $\mathcal{C}$ by the line $\overline{P_{2} P_{3}}$ we get the simple point $P_{5}=(-3,1)$, and intersecting $\mathcal{C}$ by the line $\overline{P_{2} P_{4}}$ we get the simple point $P_{6}=(5 / 6,1 / 6)$. The system $\mathcal{A}$ of adjoints of degree 3 having multiplicity 2 at the base point $P_{1}$ and 1 at the base points $P_{2}, P_{3}, P_{4}, P_{5}, P_{6}$ is defined by

$$
h(x, y, t)=-t x^{3}-3 t x^{2} y+(3 t-1) x y^{2}+(5-7 t) y^{3}+t x^{2}+x y+(7 t-5) y^{2} .
$$

Because of Bézout's theorem we expect the intersection multiplicity of curves in $\mathcal{A}$ with $\mathcal{C}$ to be $5 \cdot 3=15$. In the base points of $\mathcal{A}$ we get an intersection multiplicity of $6+2+2+2+1+1=14$. So a general element of $\mathcal{A}$ has one more intersection point with $\mathcal{C}$, depending rationally on the parameter $t$. This point traverses the curve $\mathcal{C}$ as $t$ traverses the affine line, yielding the following rational parametrization of $\mathcal{C}$ :

$$
\begin{aligned}
& x(t)=\frac{-648 t^{5}+2502 t^{4}-3900 t^{3}+3067 t^{2}-1217 t+195}{702 t^{5}-2673 t^{4}+4113 t^{3}-3196 t^{2}+1254 t-199}, \\
& y(t)=\frac{-216 t^{5}+810 t^{4}-1194 t^{3}+859 t^{2}-298 t+39}{702 t^{5}-2673 t^{4}+4113 t^{3}-3196 t^{2}+1254 t-199} .
\end{aligned}
$$


Since the simple points $P_{5}, P_{6}$ have coefficients in $\mathbb{Q}$, also the parametrization has coefficients in $\mathbb{Q}$, i.e. we need no algebraic extension of the field of coefficients.

Alternative algebraic approaches to the problem of parametrization of algebraic curves are investigated in [13] and [6]. But also in these approaches the field of coefficients $\mathbb{L}$ of the parametrization is precisely the field in which we can construct simple points of the curve $\mathcal{C}$.

So in any case, the determination of an optimal field of parametrization $\mathbb{L}$, i.e. a field achieving the bound on the extension degree in the paper of Hilbert and Hurwitz, hinges on the ability to determine points on irreducible conics. Let us demonstrate this fact by a simple example.

Example I.2: We consider the tacnode curve $\mathcal{C}$ in the affine plane over $\mathbb{C}$ defined by

$$
f(x, y)=2 x^{4}-3 x^{2} y+y^{2}-2 y^{3}+y^{4} .
$$

The tacnode curve has double points at $(0,0)$ and $(0,1)$, and one more double point in the first neighborhood of $(0,0)$. So we need one more point on $\mathcal{C}$ for determining a system of adjoints of degree 2 and dimension 1.

(a) Intersection of $\mathcal{C}$ with the line $y=-1$ yields the simple point $P_{1}=(\alpha,-1)$ on $\mathcal{C}$, where $\alpha$ is any one of the 4 roots of the irreducible polynomial $2 z^{4}+3 z^{2}+4$. This leads to the following parametrization over $\mathbb{Q}(\alpha)$ :

$$
\begin{aligned}
& x(t)=\frac{\left.-36 \alpha t^{4}+\left(48 \alpha^{2}+144\right) t^{3}+\left(108 \alpha^{3}+18 \alpha\right) t^{2}-\left(40 \alpha^{2}-84\right) t+24 \alpha^{3}+20 \alpha\right)}{4 \cdot\left(9 t^{4}+24 \alpha t^{3}-\left(16 \alpha^{2}+60\right) t^{2}-\left(24 \alpha^{3}+20 \alpha\right) t+12 \alpha^{2}+6\right)}, \\
& y(t)=\frac{-9 t^{4}-\left(18 \alpha^{3}+3 \alpha\right) t^{3}+\left(2 \alpha^{2}-33\right) t^{2}-\left(12 \alpha^{3}+2 \alpha\right) t-4}{\left(9 t^{4}+24 \alpha t^{3}-\left(16 \alpha^{2}+60\right) t^{2}-\left(24 \alpha^{3}+20 \alpha\right) t+12 \alpha^{2}+6\right)} .
\end{aligned}
$$

The coefficients of this parametrization are in $\mathbb{C} \backslash \mathbb{R}$.

(b) Intersection of $\mathcal{C}$ with the line $y=1$ yields the simple point $P_{2}=(\beta, 1)$ on $\mathcal{C}$, where $\beta$ is any one of the 2 roots of the irreducible polynomial $2 z^{2}-3$. This leads to the following parametrization over $\mathbb{Q}(\beta)$ :

$$
\begin{aligned}
x(t) & =\frac{2 \beta t^{4}+9 t^{3}-27 t-18 \beta}{11 t^{4}+24 \beta t^{3}+12 t^{2}+18}, \\
y(t) & =\frac{2 t^{4}+12 \beta t^{3}+39 t^{2}+36 \beta t+18}{11 t^{4}+24 \beta t^{3}+12 t^{2}+18} .
\end{aligned}
$$

The coefficients of this parametrization are in $\mathbb{R}$.

(c) Intersection of $\mathcal{C}$ with the line $x=1$ yields the simple point $P_{3}=(1,2)$ on

$\mathcal{C}$. This leads to the following parametrization over $\mathbb{Q}$ :

$$
\begin{aligned}
& x(t)=\frac{2 t^{4}+7 t^{3}-21 t-18}{9 t^{4}+40 t^{3}+64 t^{2}+48 t+18}, \\
& y(t)=\frac{4 t^{4}+28 t^{3}+73 t^{2}+84 t+36}{9 t^{4}+40 t^{3}+64 t^{2}+48 t+18} .
\end{aligned}
$$


Thus, the field in which we can find a simple point on $\mathcal{C}$ determines the coefficient field of the resulting parametrization.

Fig. 1

In fact, because every rational curve can be birationally transformed into a conic over the same coefficient field, it suffices to find simple points on irreducible conics.

\section{Relation of parametrization to geometric reasoning}

If in the term geometric reasoning we do not only include the process of proving or disproving geometric statements, but also the analysis and manipulation of geometric objects, then algorithms for parametrization play an important role in this wider view of geometric reasoning. Implicitization and parametrization are operations for changing the algebraic representation of geometric objects. For some operations we want implicit representations, e.g. for deciding whether a 
point actually belongs to a curve or surface. For other operations we want (some of) the geometric objects in parametric representation, e.g. for intersection of objects or for visualization.

\section{Rational points on conics}

In this section we consider irreducible conics defined over $\mathbb{Q}$, i.e. curves of degree two with rational coefficients. Such an irreducible conic in the projective plane over $\overline{\mathbb{Q}}$, the field of algebraic numbers, is defined by an irreducible homogeneous polynomial $G \in \mathbb{Q}[x, y, z]$ of degree two as the set $\left\{(\bar{x}: \bar{y}: \bar{z}) \in \mathbb{P}^{2}(\overline{\mathbb{Q}}) \mid\right.$ $G(\bar{x}, \bar{y}, \bar{z})=0\}$. In the sequel we refer to

$$
\begin{gathered}
G(x, y, z)=a x^{2}+b x y+c y^{2}+d x z+e y z+f z^{2}=0, \text { or } \\
g(x, y)=G(x, y, 1)=a x^{2}+b x y+c y^{2}+d x+e y+f=0,
\end{gathered}
$$

as the General Conic Equation. (1) defines the projective and the corresponding affine conic, respectively. We denote the projective conic by $\mathcal{C}^{*}$ and the affine conic by $\mathcal{C}$.

Definition II.1: We call $P=(\bar{x}: \bar{y}: \bar{z}) \in \mathcal{C}^{*}$ a rational point on $\mathcal{C}^{*}$ iff $P \in \mathbb{P}^{2}(\mathbb{Q})$. Analogously for the corresponding affine curve.

Our goal is to decide whether there is a rational point on the conic $\mathcal{C}^{*}$, and if so, compute one. We follow the presentation of [2], where any missing details can be found. [2] in turn is based on [4], [5] and [10].

The following theorem shows that the existence of one rational point on an irreducible conic implies that there are infinitely many rational points on it. In particular, if the projective conic $\mathcal{C}^{*}$ has a rational point, then the affine conic $\mathcal{C}$ has a rational point in $\mathbb{A}^{2}(\mathbb{Q})$. Indeed, we will basically not distinguish between $\mathcal{C}^{*}$ and $\mathcal{C}$ and treat them quite interchangeably.

Theorem II.1: An irreducible conic defined over $\mathbb{Q}$ has no or infinitely many rational points.

Proof: We give only a sketch of the proof. Suppose there is a rational point $P$ on the conic. Then we intersect the conic with a line through this rational point having a rational direction vector. We will usually get two intersection points the original rational point $P$ and an additional rational point. Varying the slope of the line leads to infinitely many other rational points on the conic.

It makes sense to distinguish between parabolas on the one hand and ellipses and hyperbolas on the other hand, since on a parabola we are guaranteed to find one (and therefore infinitely many) rational point(s). Indeed, in the parabolic case we can give a formula for a rational point on the conic (namely a rational function in the coefficients of (1)). On the other hand, on an ellipse or hyperbola we are not guaranteed that such a rational point even exists. In case it does (we 
will show how to decide that) we can compute such a point by an algorithm that is based on a constructive proof of the so called Legendre Theorem. First we deal with the parabolic case.

The parabolic case

$\mathcal{C}$ is a parabola if and only if the coefficients of (1) satisfy one of the following relations :

$$
b^{2}=4 a c, \text { or } d^{2}=4 a f, \text { or } e^{2}=4 c f .
$$

W.l.o.g. we assume now the case $b^{2}=4 a c$, i.e. we consider a parabola with respect to $x$ and $y$, whereas $z$ is the homogenizing variable.

First, let us assume $c \neq 0$. By simple expansion we have

$$
4 c g(x, y)=(b x+2 c y+e)^{2}+d^{\prime} x+f^{\prime},
$$

where $d^{\prime}=4 c d-2 b e, f^{\prime}=4 c f-e^{2}$. Because $\mathcal{C}$ is irreducible, we have $d^{\prime} \neq 0$. Thus, a rational solution is given by

$$
\bar{x}=-\frac{f^{\prime}}{d^{\prime}}, \bar{y}=-\frac{e+b \bar{x}}{2 c},(\bar{z}=1) .
$$

Now the remaining case to treat is $c=0$. Again by irreducibility, we have $a \neq 0$. A rational solution is then given by

$$
\bar{x}=-\frac{d+b \bar{y}}{2 a}, \bar{y}=-\frac{f^{\prime}}{d^{\prime}},(\bar{z}=1),
$$

where $d^{\prime}=4 a e-2 b d$ and $f^{\prime}=4 a f-d^{2}$.

Example II.1: Consider the parabola defined by

$$
g(x, y)=x^{2}+2 x y+y^{2}+x+2 y-2,
$$

i.e. $(a, b, c, d, e, f)=(1,2,1,1,2,-2)$. Since $a \neq 0$ and $c \neq 0$, we might use both formulae. Let us first use the formula for the case $c \neq 0$ :

$$
d^{\prime}=4 c d-2 b e=-4, \text { and } f^{\prime}=4 c f-e^{2}=-12 .
$$

So we get the rational point

$$
\bar{x}=-\frac{f^{\prime}}{d^{\prime}}=-3, \quad \bar{y}=-\frac{e+b \bar{x}}{2 c}=2
$$

on the parabola.

Now we use the formula for the case $a \neq 0$ :

$$
d^{\prime}=4 a e-2 b d=4 \text {, and } f^{\prime}=4 a f-d^{2}=-9 .
$$

So we get the rational point

$$
\bar{x}=-\frac{d+b \bar{y}}{2 a}=-\frac{11}{4}, \quad \bar{y}=-\frac{f^{\prime}}{d^{\prime}}=\frac{9}{4}
$$


on the parabola.

The hyperbolic/elliptic case

We consider (1), but we impose other conditions on the coefficients. We use ideas from [5]. The hyperbolic/elliptic case is characterized by

$$
b^{2} \neq 4 a c \text { and } d^{2} \neq 4 a f \text { and } e^{2} \neq 4 c f .
$$

We consider the dehomogenization with respect to $z$ (i.e. in what follows, we will only make use of $b^{2} \neq 4 a c$ ). Let us define

$$
\begin{gathered}
N=4 d e-4 b f, D=4 a c-b^{2}, \\
M_{1}=4 c^{2} d^{2}-4 b c d e+4 a c e^{2}+4 b^{2} c f-16 a c^{2} f, \\
M_{2}=4 a^{2} e^{2}-4 b a d e+4 a c d^{2}+4 b^{2} a f-16 c a^{2} f .
\end{gathered}
$$

We consider two cases.

(CASE $a=c=0$ ) In this case we have $b \neq 0$ and $N \neq 0$ (by irreducibility). In the new coordinates

$$
\begin{aligned}
x^{\prime} & =b(x+y)+d+e, \\
y^{\prime} & =b(x-y)-d+e
\end{aligned}
$$

the equation $4 b g(x, y)=0$ has the following form :

$$
\left(x^{\prime}\right)^{2}-\left(y^{\prime}\right)^{2}=N \text {. }
$$

(CASE $c \neq 0)$ We have $M_{1} \neq 0$ and $\left(D>0 \Rightarrow M_{1}>0\right)$ (both conditions are consequences of irreducibility). Under the coordinate change

$$
\begin{gathered}
x^{\prime}=D x+2 d c-b e, \\
y^{\prime}=b x+2 c y+e
\end{gathered}
$$

the equation $4 c D g(x, y)=0$ becomes

$$
\left(x^{\prime}\right)^{2}+D\left(y^{\prime}\right)^{2}=M_{1} .
$$

The case $a \neq 0$ is totally analogous to the case $c \neq 0$ (just interchange the roles of $x$ and $y$ and therefore also those of $a$ and $c$ and those of $d$ and $e$; in addition use $M_{2}$ instead of $M_{1}$ ).

In both cases we arrive at an equation of the form

$$
X^{2}+K Y^{2}=L,
$$

where $K, L \in \mathbb{Q}$, and in both cases we do not have $(K>0 \wedge L<0)$, which would exclude the existence of a real solution.

Hence we can restrict us to equations of this form . Switching to homogeneous coordinates we set

$$
X=\frac{x}{z}, Y=\frac{y}{z}, K=\frac{b^{\prime}}{a^{\prime}}, L=-\frac{c^{\prime}}{a^{\prime}}
$$


Note that if $K=k_{1} / k_{2}, L=l_{1} / l_{2}$ we may choose $a^{\prime}=\operatorname{lcm}\left(k_{2}, l_{2}\right)$, $b^{\prime}=k_{1} l_{2} / \operatorname{gcd}\left(k_{2}, l_{2}\right)$, and $c^{\prime}=-l_{1} k_{2} / \operatorname{gcd}\left(k_{2}, l_{2}\right)$. Then $(2)$ becomes the diophantine equation

$$
a^{\prime} x^{2}+b^{\prime} y^{2}+c^{\prime} z^{2}=0 .
$$

Clearly $a^{\prime}, b^{\prime}$, and $c^{\prime}$ are nonzero and do not all have the same sign (look at their definitions and use $\neg(K>0 \wedge L<0))$. But we want to achieve more, namely the reduction of (3) to an equation of similar form whose coefficients are squarefree and pairwise relatively prime. We use ideas from [10]. Let us assume that

$$
a^{\prime}=a_{1}^{\prime} r_{1}^{2}, b^{\prime}=b_{1}^{\prime} r_{2}^{2}, c^{\prime}=c_{1}^{\prime} r_{3}^{2},
$$

where $a_{1}^{\prime}, b_{1}^{\prime}$, and $c_{1}^{\prime}$ are squarefree ${ }^{2}$. Consider

$$
a_{1}^{\prime} x^{2}+b_{1}^{\prime} y^{2}+c_{1}^{\prime} z^{2}=0 .
$$

(4) has an integral solution iff (3) has one.

Now, we divide (4) by $\operatorname{gcd}\left(a_{1}^{\prime}, b_{1}^{\prime}, c_{1}^{\prime}\right)$, getting

$$
a^{\prime \prime} x^{2}+b^{\prime \prime} y^{2}+c^{\prime \prime} z^{2}=0 .
$$

What remains is to make the coefficients pairwise relatively prime.

Let $g_{1}=\operatorname{gcd}\left(a^{\prime \prime}, b^{\prime \prime}\right), a^{\prime \prime \prime}=a^{\prime \prime} / g_{1}, b^{\prime \prime \prime}=b^{\prime \prime} / g_{1}$, and let $(\bar{x}, \bar{y}, \bar{z})$ be an integral solution of (5). Then $g_{1} \mid c^{\prime \prime} \bar{z}^{2}$, and hence, since $\operatorname{gcd}\left(a^{\prime \prime}, b^{\prime \prime}, c^{\prime \prime}\right)=1$, we have $g_{1} \mid \bar{z}^{2}$. Furthermore, since $g_{1}$ is squarefree (since $a^{\prime \prime}, b^{\prime \prime}$ are), we have $g_{1} \mid \bar{z}$. So, letting $z=g_{1} z^{\prime}$ and cancelling (5) by $g_{1}$, we arrive at

$$
a^{\prime \prime \prime} x^{2}+b^{\prime \prime \prime} y^{2}+\underbrace{c^{\prime \prime} g_{1}}_{c \prime \prime \prime \prime}\left(z^{\prime}\right)^{2}=0 .
$$

We have $\operatorname{gcd}\left(a^{\prime \prime \prime}, b^{\prime \prime \prime}\right)=1$ and $c^{\prime \prime \prime}$ is squarefree since $g_{1}$ and $c^{\prime \prime}$ are relatively prime. Repeating this process with $g_{2}=\operatorname{gcd}\left(a^{\prime \prime \prime}, c^{\prime \prime \prime}\right)$ and $g_{3}=\operatorname{gcd}\left(b^{\prime \prime \prime \prime}, c^{\prime \prime \prime \prime}\right)$ we arrive at

$$
a\left(x^{\prime}\right)^{2}+b\left(y^{\prime}\right)^{2}+c\left(z^{\prime}\right)^{2}=0
$$

the so called Legendre Equation. We note $: a, b$, and $c$ are nonzero, do not all have the same sign, are squarefree, and pairwise relatively prime. We will now try to find an integral solution of this diophantine equation that can then be

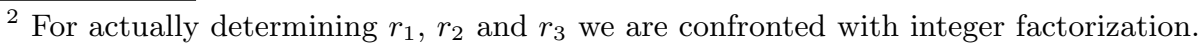
Although there are no polynomial-time algorithms known for the factorization of large integers (the most powerful general purpose factoring method leads to a factorization of an integer $m$ in time $O[\exp (2 L\{L[L(m)]\})]$, where $L(m)$ denotes the length of $m$ ) this does not lead to problems in practical computations. Usually the integers to be factored are small enough such that succesful and fast application of integer factorization commands as provided by computer algebra systems is guaranteed.

Things are trivial if $a^{\prime}, b^{\prime}$, and $c^{\prime}$ are polynomials (this will occur if we deal with conic equations over $\mathbb{Q}(t)$ as in the following section) since squarefree factorization of polynomials poses no problems at all.
} 
transformed back to a rational solution of the original equation. Algorithmic formulations (in pseudocode) of the above steps (including the parabolic case) can be found in [2], where in the appendix one can also find a Maple implementation.

Hence the problem of finding a rational point on an ellipse/ hyperbola reduces to the problem of finding a nontrivial integral solution of the so called Legendre Equation

$$
a x^{2}+b y^{2}+c z^{2}=0
$$

where $a, b$, and $c$ are integers such that $a b c \neq 0$. By a nontrivial integral solution we mean a solution $(\bar{x}, \bar{y}, \bar{z}) \in \mathbb{Z}^{3}$ with $(\bar{x}, \bar{y}, \bar{z}) \neq(0,0,0)$ and $\operatorname{gcd}(\bar{x}, \bar{y}, \bar{z})=1$. We also pointed out that we may assume, w. l. o. g.,

$$
\begin{aligned}
a> & 0, b<0 \text { and } c<0, \\
& a, b, \text { and } c \text { are squarefree, } \\
\operatorname{gcd}(a, b)= & \operatorname{gcd}(a, c)=\operatorname{gcd}(b, c)=1 .
\end{aligned}
$$

We now deal with necessary and sufficient conditions in order that (8) has nontrivial integral solutions. Such conditions are given by the Theorem of Legendre. For a formulation of Legendre's Theorem we need the notion of quadratical residues.

Definition II.1: Let $m, n$ be nonzero integers. Then $m$ is a quadratic residue modulo $n$ (written $m R n$ ) iff $\exists x \in \mathbb{Z}: x^{2} \equiv_{n} m$.

Now we can state the theorem.

Theorem II.3: (Legendre, Version 1) Suppose $a, b$, and $c$ satisfy (9), (10) and (11). Then (8) has a nontrivial integral solution iff

$$
-a b R c,-b c R a, \text { and }-a c R b .
$$

We prove only the necessity of (12) for this first version of the theorem and prove the sufficiency then for a second (equivalent) version.

Proof: (Legendre's Theorem, necessity of (12))

Let $(\bar{x}, \bar{y}, \bar{z})$ be a solution of $(8)$; it follows that $\operatorname{gcd}(c, \bar{x})=1$. For if any prime $p$ divides $\operatorname{gcd}(c, \bar{x})$, then $p$ divides $b \bar{y}^{2}$ but $p$ does not divide $b(\operatorname{since} \operatorname{gcd}(b, c)=1$ by (11)) and so $p$ divides $\bar{y}$. Consequently we have $p^{2}$ divides $a \bar{x}^{2}+b \bar{y}^{2}$ and hence $p^{2}$ divides $c \bar{z}^{2}$. But $c$ is squarefree and so $p$ divides $\bar{z}$. This contradicts the assumption $\operatorname{gcd}(\bar{x}, \bar{y}, \bar{z})=1$.

As $\operatorname{gcd}(c, \bar{x})=1$ we can find $\bar{x}^{\prime}$ satisfying $\overline{x x}^{\prime} \equiv_{c} 1$. Also, clearly

$$
a \bar{x}^{2}+b \bar{y}^{2} \equiv_{c} 0,
$$

and so, by multiplying with $b\left(\bar{x}^{\prime}\right)^{2}$,

$$
b^{2}\left(\bar{x}^{\prime}\right)^{2} \bar{y}^{2} \equiv_{c}-a b\left(\overline{x x}^{\prime}\right)^{2} \equiv_{c}-a b .
$$


Thus $-a b R c$ holds. The remaining conditions can be derived similarly.

Theorem II.4: (Legendre, Version 2) Let $a$ and $b$ be positive squarefree integers. Then

$$
a x^{2}+b y^{2}=z^{2}
$$

has a nontrivial solution if and only if the following three conditions are satisfied:

$$
\begin{aligned}
& a R b, \\
& b R a, \\
& -\frac{a b}{\operatorname{gcd}(a, b)^{2}} R \operatorname{gcd}(a, b) .
\end{aligned}
$$

The equivalence of these two versions is easily established (see [2]). The following constructive proof of the Legendre Equation can be found in [4], we give the presentation from [2] (where all missing details can be found).

Proof: (Theorem II.4) The necessity of (14) to (16) is established by the necessity of (12) for the solvability of (8) and the claimed equivalence of the two versions of Legendres Theorem. So we show sufficiency and hence assume now that (14) to (16) hold.

Let us first of all consider two special (simple) instances of (13)

(CASE $a=1)$ Obviously, $(\bar{x}, \bar{y}, \bar{z})=(1,0,1)$ is a solution.

(CASE $a=b$ ) Condition (16) requires -1 to be a square modulo $b$. If this is the case, we can find integers $r$ and $s$ such that $b=r^{2}+s^{2}$ (consider this as an easy lemma), leading to a solution $(\bar{x}, \bar{y}, \bar{z})=\left(r, s, r^{2}+s^{2}\right)$.

Now we proceed to the general case. We may assume $a>b$, for if $b>a$ just interchange the roles of $x$ and $y$. The strategy will be the following: We construct a new form $A x^{2}+b y^{2}=z^{2}$ satisfying the same hypotheses as (13), $0<A<a$, and having a nontrivial solution iff (13) does so (and a solution of (13) can be computed from a solution of the new form). After a finite number of steps, interchanging $A$ and $b$ in case $A$ is less than $b$, we arrive at one of the cases $A=1$ or $A=b$, each of which has been settled. Now for the details.

We assume now that (14) - (16) hold. By (15) there exist integers $x$ and $k$ such that

$$
x^{2}=b+k a .
$$

Let $k=A m^{2}$, where $A$ is the squarefree part of $k$. Also note that we can choose $x$ such that $|x| \leq a / 2$ by choosing the absolute least residue of $x$ modulo $a$ ("symmetric representation of the integers modulo $a$ "). Let us now restate (17) as

$$
x^{2}=b+A m^{2} a .
$$

First of all we show that $0<A<a$. Since by (18) and $b<a$

$$
0 \leq x^{2}=b+A m^{2} a<a+A m^{2} a=a\left(1+A m^{2}\right)
$$


we have $0<1+A m^{2}$, and hence $A \geq 0$. But if $A=0$, then (18) gives $x^{2}=b$, contradicting the fact that $b$ is squarefree. So we established $A>0$. On the other hand by (18) and $b>0$ and since $|x| \leq a / 2$ we have

$$
A m^{2} a<x^{2} \leq \frac{a^{2}}{4},
$$

and so we have $A \leq A m^{2}<a / 4(<a)$. So we consider now

$$
A x^{2}+b Y^{2}=Z^{2} \text {. }
$$

Clearly $A, b$ are positive and squarefree integers. So we want to show

$$
\begin{aligned}
& A R b, \\
& b R A, \\
& -\frac{A b}{\operatorname{gcd}(A, b)^{2}} R \operatorname{gcd}(A, b) .
\end{aligned}
$$

In addition, we need that (13) has a nontrivial solution if and only if (19) has one, which will be shown constructively.

(Show (21)) With $g=\operatorname{gcd}(a, b)$, let $b_{1}=b / g, a_{1}=a / g$. We show $A R g$ and $A R b_{1}$. Then, we have $A R b_{1} g$ (consider this as a lemma), i.e. $A R b$. First of all, note that (18) may be written as

$$
x^{2}=b_{1} g+A m^{2} a_{1} g .
$$

Since $g$ is squarefree we have that $g$ divides $x$. Setting $x_{1}=\frac{x}{g}$ and cancelling gives

$$
g x_{1}^{2}=b_{1}+A m^{2} a_{1} .
$$

Thus $A m^{2} a_{1} \equiv_{g}-b_{1}$, and hence

$$
A m^{2} a_{1}^{2} \equiv_{g}-a_{1} b_{1} .
$$

Also note that $\operatorname{gcd}(m, g)=1$, since a common factor would divide $b_{1}$ (by (24)) and hence $b=b_{1} g$ would not be squarefree. But also $\operatorname{gcd}\left(a_{1}, g\right)=1$ since $a=a_{1} g$ is squarefree. Let $m^{\prime}$ and $a_{1}^{\prime}$ be the inverses of $m$ respectively $a_{1}$ modulo $g$. By (16) (i.e. by $-a_{1} b_{1} R g$ ) we may choose $y$ such that $y^{2} \equiv_{g}$ $-a_{1} b_{1}$. Now $(25)$ becomes $A \equiv_{g}\left(m^{\prime}\right)^{2}\left(a_{1}^{\prime}\right)^{2} y^{2}$, i.e. $A R g$. So this part is done. It remains to show $A R b_{1}$.

By (23) we have

$$
x^{2} \equiv_{b_{1}} A m^{2} a .
$$

By (14) (i.e. by $a R b)$ we have $a R b_{1}$. Note also that $\operatorname{gcd}\left(a, b_{1}\right)=1$ since a common factor would divide $b_{1}$ and $g$, contradicting the fact that $b=b_{1} g$ is squarefree. Similarly, $\operatorname{gcd}\left(m, b_{1}\right)=1$ (use $\left.(23)\right)$. Let $a^{*}$ and $m^{*}$ be the inverses of $a$ respectively $m$ modulo $b_{1}$. Let $z$ be such that $z^{2} \equiv_{b_{1}} a$ and let $z^{*}$ be its inverse modulo $b_{1}$. Now (26) becomes

$$
A \equiv_{b_{1}} x^{2}\left(m^{*}\right)^{2} a^{*} \equiv_{b_{1}} x^{2}\left(m^{*}\right)^{2}\left(z^{*}\right)^{2},
$$

i.e. $A R b_{1}$. 
(Show (22)) By (18), we have $b R A$ immediately.

(Show (23)) With $r=\operatorname{gcd}(A, b)$ let $A_{1}=A / r, b_{2}=b / r$. We have to show $-A_{1} b_{2} R r$.

From (18) we conclude

$$
x^{2}=b_{2} r+A_{1} r m^{2} a .
$$

Since $r$ is squarefree we have $r$ divides $x$. So

$$
\begin{gathered}
A_{1} m^{2} a \equiv-b_{2} \quad(\bmod r), \text { or } \\
-A_{1} b_{2} m^{2} a \equiv b_{2}^{2} \quad(\bmod r) .
\end{gathered}
$$

Since $\operatorname{gcd}(a, r)=\operatorname{gcd}(m, r)=1$, we may choose $a^{+}$and $m^{+}$as the inverses of $a$ respectively $m$ modulo $r$. Furthermore, from (14) (i.e. from $a R b$ ) we obtain $a R r$. Choose $w$ such that $w^{2} \equiv_{r} a$. Denote by $w^{+}$the inverse of $w$ modulo $r$. Then (27) becomes

$$
-A_{1} b_{2} \equiv_{r} b_{2}^{2}\left(m^{+}\right)^{2} a^{+} \equiv_{r} b_{2}^{2}\left(m^{+}\right)^{2}\left(w^{+}\right)^{2},
$$

i.e. $-A_{1} b_{2} R r$.

So we established (20) - (22) for (19). Assume now that (19) has a nontrivial solution $(\bar{X}, \bar{Y}, \bar{Z})$. Then

$$
A \bar{X}^{2}=\bar{Z}^{2}-b \bar{Y}^{2}
$$

Multiplying this by (18) (i.e. by $A m^{2} a=x^{2}-b$ ) gives

$$
\begin{aligned}
a(A \bar{X} m)^{2} & =\left(\bar{Z}^{2}-b \bar{Y}^{2}\right)\left(x^{2}-b\right)= \\
& =(\bar{Z} x+b \bar{Y})^{2}-b(x \bar{Y}+\bar{Z})^{2} .
\end{aligned}
$$

Thus a solution of (13) is

$$
\bar{x}=A \bar{X} m, \bar{y}=x \bar{Y}+\bar{Z}, \bar{z}=\bar{Z} x+b \bar{Y} .
$$

Written in matrix notation we have

$$
\left[\begin{array}{l}
\bar{x} \\
\bar{y} \\
\bar{z}
\end{array}\right]=\left[\begin{array}{ccc}
A m & 0 & 0 \\
0 & x & 1 \\
0 & b & x
\end{array}\right] \cdot\left[\begin{array}{l}
\bar{X} \\
\bar{Y} \\
\bar{Z}
\end{array}\right] .
$$

The matrix is invertible since its two blocks are : the second $(2 \times 2)$ block has determinant $x^{2}-b \neq 0$ (since $b$ is squarefree). The solution is nontrivial since we claim that $\bar{x}=A m \bar{X} \neq 0$. Suppose $A m=0$. Then by (18) we have $x^{2}=b$, contradicting the squarefreeness of $b$. Suppose $\bar{X}=0$. Then by (28) we have $\bar{Z}^{2}=b \bar{Y}^{2}$, contradicting the squarefreeness of $b$.

Algorithm for solving the Legendre Equation

The constructive proof for the existence of a nontrivial integral solution of the Legendre Equation in the previous section leads to a recursive algorithm 
for computing such a solution. We will give a formulation in a Pascal-like pseudocode. We do not consider an algorithmic formulation of the transformations that lead from the General Conic Equation to the Legendre Equation, see [2] for that purpose. We assume the procedure msqrt ("modular squareroot"), that has the following meaning : for integers $a, b$ with $a R b$ we have

$$
\operatorname{msqrt}(a, b)^{2} \equiv_{b} a \text {. }
$$

Such a procedure exists for example in Maple ${ }^{\mathrm{TM}}$. We work in symmetric representation of the integers modulo any number. In addition, we use the knowledge that for a natural number $r$ with $-1 R r$ there exist integers $x, y$ such that $r=x^{2}+y^{2}$ (for a proof of that fact and a procedure Circle that computes such $x$ and $y$ for given $r$ see again [2]). Finally we need a procedure sqfrp ("squarefree part") for computing the squarefree part of an integer, i.e. for $n=\prod_{p \text { prime }} p^{n_{p}}$ we have

$$
\operatorname{sqfrp}(n)=\prod_{p \text { prime }} p^{\bmod \left(n_{p}, 2\right)} .
$$

Now we can give the pseudocode.

PROC LegendreSolve $(\downarrow a \downarrow b \uparrow x \uparrow y \uparrow z)$

IN :

$a, b \in \mathbb{Z}:$

positive, squarefree with $a R b, b R a,-a b / \operatorname{gcd}(a, b)^{2} R \operatorname{gcd}(a, b)$.

\section{OUT :}

$x, y, z \in \mathbb{Z}$

such that $a x^{2}+b y^{2}=z^{2}$.

\section{LOCAL}

$r, s, T, A, B, X, Y, Z, m \in \mathbb{Z}$

BEGIN

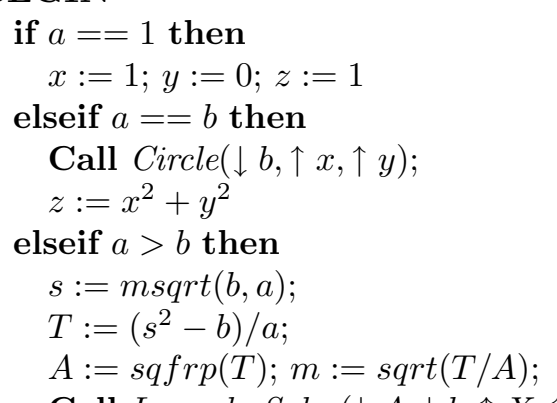

Call LegendreSolve $(\downarrow A, \downarrow b, \uparrow X, \uparrow Y, \uparrow Z)$;

$x:=A X m ; y:=s Y+Z ; z:=s Z+b Y$

else

$s:=\operatorname{msqrt}(a, b)$

$T:=\left(s^{2}-a\right) / b$

$B:=\operatorname{sqfrp}(T) ; m:=\operatorname{sqrt}(T / B)$;

Call LegendreSolve $(\downarrow B, \downarrow a, \uparrow Y, \uparrow X, \uparrow Z)$; 


\section{$y:=B Y m ; x:=s X+Z ; z:=s Z+a X$ \\ end if \\ END LegendreSolve}

Some words on the number of self-references in LegendreSolve. The worst thing that can happen is that we reduce both coefficients of

$$
a x^{2}+b y^{2}=z^{2}
$$

to 1. The number of self-references of LegendreSolve needed to achieve this is bounded by $2 \log _{4}(\max (a, b))$, since every time we reduce a coefficient, it is reduced by a factor of 4 at least. In the situation $a=b$ we call Circle (and no more call to LegendreSolve is needed), which calls itself not more than $\log (a)$ times (compare [2]). So in all cases, the maximal number of any procedure calls is $O(\log (\max (a, b))$. The (theoretical) time complexity of the main algorithm (input an irreducible conic over the rational numbers and output a rational point if existent) would be at least exponential in any way since we use integer factorization in the implementation of the procedure sqfrp. But this fact has not turned out to be an obstacle in practical computations.

Some words on the space complexity of the main algorithm: If we denote by $l$ the maximum length of any numerator or denominator of the coefficients of the General Conic Equation, then we have for the (integer) coefficients $a$ and $b$ of the associated Special Legendre Equation

$$
a x^{2}+b y^{2}=z^{2}
$$

that $^{3}$

$$
L(\max (a, b)) \leq 12 l .
$$

(This worst case bound may be reached if the numerators and denominators of the General Conic Equation are all of equal length). For the diophantine solution $(\bar{x}, \bar{y})$ of the Special Legendre Equation we can give the bound

$$
L(\max (\bar{x}, \bar{y})) \leq 5 L(\max (a, b))^{2} .
$$

Concluding we obtain (the backward transformations do not influence the order) that the maximal length of any numerator or denominator for the (rational) solution of the General Conic Equation is $O\left(l^{2}\right)$.

Example II.2: Consider the conic defined by

$$
g(x, y)=x^{2}-4 x y-3 y^{2}+4 x+8 y-5 .
$$

Carrying out the above described transformations leads to the corresponding (Special) Legendre Equation

$$
7 x^{2}+21 z^{2}=y^{2}
$$

\footnotetext{
${ }^{3}$ We assume integers in decimal representation.
} 
Now we call LegendreSolve $(\downarrow 7, \downarrow 21, \uparrow x, \uparrow z, \uparrow y)$. Here is a trace of the corresponding values of the local variables $s, T, B, m$ and the recursive calls of LegendreSolve :

$$
\begin{aligned}
& (s, T, B, m)=(7,2,2,1) ; \text { CallLegendreSolve }(\downarrow 2, \downarrow 7, \uparrow Y, \uparrow X, \uparrow Z) ; \\
& (s, T, B, m)=(3,1,1,1) ; \text { CallLegendreSolve }(\downarrow 1, \downarrow 2, \uparrow Y, \uparrow X, \uparrow Z) ;
\end{aligned}
$$

Now the equation $Y^{2}+2 X^{2}=Z^{2}$ has the integral solution $(Y, X, Z)=(1,0,1)$. So the procedure produces the following integral solutions of the stacked equations :

$$
\begin{aligned}
(X, Y, Z) & =(1,1,3), \\
(z, x, y) & =(2,10,28),
\end{aligned}
$$

the latter being an integral solution of $7 x^{2}+21 z^{2}=y^{2}$, the (Special) Legendre Equation. Inverting the above indicated transformations we arrive at a rational solution of $g(x, y)=0$ :

$$
(x, y)=\left(-\frac{3}{7}, \frac{16}{7}\right)
$$

Real points on conics $^{4}$

Let us assume that no rational point lies on the conic. In this case we ask whether there is at least a real point on the conic, i.e. whether there exists $(\bar{x}, \bar{y}) \in \mathbb{R}^{2}$ such that

$$
g(\bar{x}, \bar{y})=0 .
$$

Since every parabola contains a rational point, we only have to consider the elliptic/hyperbolic case. Again we transform the General Conic Equation to an equation of the form

$$
x^{2}+K y^{2}=L,
$$

where $K, L$ are rational numbers. A real point on the conic exists if and only if $\neg(K \geq 0 \wedge L<0)$. In this case, a real solution of (29) is given by

$$
\begin{aligned}
& (\bar{x}, \bar{y})=(\sqrt{L}, 0) \quad \text { if } \quad L>0, \\
& (\bar{x}, \bar{y})=\left(0, \sqrt{\frac{L}{K}}\right) \quad \text { if } \quad L<0 .
\end{aligned}
$$

By back transformation, we arrive at a real solution for the General Conic Equation.

\footnotetext{
${ }^{4}$ The question when a rational algebraic plane curve over $\mathbf{Q}$ is parametrizable over $\mathbf{R}$ is treated in section 3.3 ("Parametrizing over the reals") of [12]. We state here the main result.

Theorem $\mathbf{3 . 2}$ (in [12]) A rational algebraic plane curve over $\mathbf{Q}$ is parametrizable over $\mathbf{R}$ if and only if it is not birationally equivalent over $\mathbf{R}$ to the conic $x^{2}+y^{2}+z^{2}$.
} 


\section{Points on conics over the rational function field}

As in the rational case, we only have to consider the reduced equation

$$
X^{2}+K(t) Y^{2}=L(t),
$$

where $K, L \in \mathbb{Q}(t)$. Our goal is to find rational functions $X(t), Y(t)$ satisfying (30). This solves the problem of finding rational functions satisfying the General Conic Equation with coefficients in $\mathbb{Q}(t)$ completely. For solving (30), we try to exploit the method used for the rational case. In order to point out the analogy between these cases, we note that $\mathbb{Q}(t)$ is the quotient field of $\mathbb{Q}[t]$, a Euclidean Domain (ED for short), like $\mathbb{Q}$ is the quotient field of $\mathbb{Z}$ (the standard example of an ED). This means that we can make use of modular arithmetic, as we did in the rational case. Also those details of the rational case depending on factorization can be adapted, since every ED is a Unique Factorization Domain (UFD). So we can do all transformations that we did in the rational case and finally arrive at an equation of the form

$$
a(t) x^{2}+b(t) y^{2}=z^{2},
$$

where $a$ and $b$ are nonzero and squarefree polynomials satisfying (at least if there exists a rational solution)

$$
\begin{aligned}
& a R b, \\
& b R a, \\
& -\frac{a b}{\operatorname{gcd}(a, b)^{2}} R \operatorname{gcd}(a, b) .
\end{aligned}
$$

(The notion of quadratic residue for polynomials is analogous to the one for integers). W. l. o. g. let us assume $\operatorname{deg}(a) \geq \operatorname{deg}(b)$. From the proof of Legendre's Theorem for the rational case we know that in the new coordinates

$$
\begin{aligned}
& x=A X m, \\
& y=s Y+Z, \\
& z=s Z+b Y,
\end{aligned}
$$

where $^{5}$

$$
\begin{aligned}
s(t) & =\text { pmsqrt }(b(t), a(t)), \\
k(t) & =\frac{s(t)^{2}-b(t)}{a(t)}, \\
A(t) & =\operatorname{sqfrp}(k(t)), \\
m(t) & =\sqrt{\frac{k(t)}{A(t)}}
\end{aligned}
$$

${ }^{5}$ pmsqrt: "polynomial modular squareroot", i. e.

$$
\operatorname{pmsqrt}(b(t), a(t))^{2}=b(t) \bmod a(t) .
$$


(31) has the form

$$
A X^{2}+b Y^{2}=Z^{2}
$$

In analogy to the rational case $A$ is smaller than $a$ in some sense : in the rational case it was the absolute value of $a$ that dropped; here it is the degree of the polynomial $a(t)$ that drops. The point now is that by iterated application of the above transformation (as in the rational case) we arrive at some simple instances of the (polynomial) Legendre Equation, where we can decide the existence of a rational (function) solution and - if one exists - give a solution. Technical details and algorithmic formulations can be found in [2].

The problem treated in this chapter arises in the context of parametrizing surfaces over $\mathbb{Q}$. In particular, the following two problems are closely related :

1. Parametrize a conic $f(x, y)=0$ (where $f \in \mathbb{Q}(t)[x, y]$ ) with rational functions in $s$ and coefficients in $\mathbb{Q}(t)$.

2. Parametrize a surface $F(x, y, t)=0$ (where $F \in \mathbb{Q}[x, y, t]$ is of total degree 2 in $x$ and $y$ ) with rational functions in $s$ and $t$.

The exact relationship and the application of our results to the parametrization of such surfaces needs to be investigated further.

\section{Conclusion}

Given an algebraic curve defined over the rational number field, we can decide whether the curve has genus 0 and infinitely many points over the rational numbers and therefore can be parametrized over the rationals. Similarly we can decide whether a real curve can be parametrized over the reals. We are able to extend these decision methods from $\mathbb{Q}$ to $\mathbb{Q}(t)$, the field of rational functions over $\mathbb{Q}$. We conjecture that this extension should lead to parametrization algorithms for certain surfaces of interest in computer-aided-geometric-design, such as discussed in [9]. This needs further investigation.

\section{References}

1. Hilbert, D., Hurwitz, A.: Über die Diophantischen Gleichungen vom Geschlecht Null. Acta math. 14 (1890) 217-224

2. Hillgarter, E.: Rational Points on Conics. Diploma Thesis, RISC-Linz, J. Kepler Universität Linz, Austria (1996)

3. Hoschek, J., Lasser, D.: Fundamentals of Computer Aided Geometric Design, A.K. Peters, Wellesley MA (1993)

4. Ireland, K., Rosen, M.: A Classical Introduction to Modern Number Theory, Springer Verlag, New York Heidelberg Berlin (1982)

5. Krätzel, E.: Zahlentheorie, VEB Dt. Verlag der Wissenschaften, Berlin (1981) 
6. Mňuk, M.: Algebraic and Geometric Approach to Parametrization of Rational Curves, Ph.D. Dissertation, RISC-Linz, J. Kepler Universität Linz, Austria (1995)

7. Mňuk, M., Sendra, J.R., Winkler, F.: On the Complexity of Parametrizing Curves. Beiträge zur Algebra und Geometrie 37/2 (1996) 309-328

8. Nielson, G.M.: Cagd's Top Ten: What to Watch. IEEE Computer Graphics \& Applications (Jan. 1993)

9. Peternell, M., Pottmann, H.: Computing Rational Parametrizations of Canal Surfaces. J. Symbolic Comp. 23/2\&3 (1996) 255-266

10. Rose, H.E.: A Course in Number Theory, Clarendon Press, Oxford (1988)

11. Sendra, J.R., Winkler, F.: Symbolic Parametrization of Curves. J. Symbolic Comp. 12 (1991) 607-631

12. Sendra, J.R., Winkler, F.: Parametrization of Algebraic Curves over Optimal Field Extensions. J. Symbolic Comp. 23/2\&3 (1996) 191-207

13. van Hoeij, M.: Rational Parametrization of Algebraic Curves using a Canonical Divisor. J. Symbolic Comp. 23/2\&3 (1996) 209-227

14. Walker, R.J.: Algebraic Curves, Princeton University Press (1950)

This article was processed using the IATEX macro package with LLNCS style 